\title{
Semantic and morphological cross-word priming during sentence reading
}

\author{
Katarina Marjanovič ${ }^{\mathrm{a}, *}$, Davide Crepaldi ${ }^{\mathrm{a}}$ \\ ${ }^{a}$ International School for Advanced Studies (SISSA), Trieste, Italy
}

\begin{abstract}
Our current understanding of visual word identification is difficult to extend to text readingboth experiments and theories focus primarily, if not exclusively, on out-of-context individual words. Here, we try to fill this gap by studying cross-word semantic and morphological priming within sentences in a natural reading, eye tracking experiment. We find that words are skipped more when they are preceded in the sentence by semantically related primes. Also, cross-word semantic priming manifests itself in later (e.g., gaze duration), but not in earlier (e.g., first-of-many fixations) indexes of eye movement on the target words. We also find that semantic priming is not modulated by the morphological agreement between primes and targets; and that morphological agreement does not yield any priming per se. These results point to independent lexical-semantic and morphological processing during sentence reading, and suggest cross-word reset for the latter, but not for the former.
\end{abstract}

Keywords: natural sentence reading, eye tracking, semantic priming, morphological priming, cross--word priming

\section{Introduction}

Over the last decades, we have learned a great deal about visual word identification (e.g., Adelman et al., 2010; Forster and Veres, 1998; Marelli et al., 2015; Rastle et al., 2000; Xu and Taft, 2014). Our knowledge is so rich and articulated that it fostered the construction of fully-fledged computational models - our theories of visual word processing have reached a mechanistic level (e.g., Adelman, 2011; Coltheart et al., 2001; Davis, 2010; Perry et al., 2010). This knowledge, however, is very much focused on individual word identification, while our everyday life experience is dominated by text reading instead. This requires the visual identification system to deal with several words in a very short amount of time ( 300 words per minute; Pelli et al., 2007) - the very detailed models described above remain agnostic as to this fundamental aspect of human reading.

\footnotetext{
${ }^{*}$ Corresponding author

Email addresses: katarina.marjanovic@sissa.it (Katarina Marjanovič), davide.crepaldi@sissa.it (Davide Crepaldi)
} 
From this perspective, models of eye movement are a particularly interesting case (Engbert et al., 2005; Reichle et al., 2006). By design, they have to deal with the identification of several individual words during sentence reading; that is, they face the problem of having the visual word identification system engaged with several words in very rapid succession (in serial models, such as in E-Z Reader), or even simultaneously (in parallel models, such as in SWIFT). How a unique visual word identification system would deal with this issue is not obvious. More generally, these models stay clear of the lexical dynamics described in models of single word identification; they typically treat this latter process as an unarticulated whole, of which they only estimate the duration/difficulty based on proxies such as word frequency or word predictability in a given sentence context.

Of course, there are exceptions to this general trend. In their Glenmore model, Reilly and Radach (2006) include letter-level dynamics, which may allow some contact between visual word identification theories and text reading. However, the authors themselves acknowledge that they were more interested into modelling target words for saccades rather than implementing realistic lexical dynamics. The very recently released OB1-Reader, instead, makes exactly this step, and aims at integrating what we know about individual word identification into a general account of text reading (Snell et al., 2018). Here we follow very much in the same spirit, moving the focus from eye movements, which is the main target in OB1-Reader, to post-orthographic processing levels in lexical identification, and how these are affected by the engagement of the visual word identification system with multiple words in very rapid succession.

Our starting point is one of the most established phenomena in the individual word identification literature, namely, priming. It is extremely well demonstrated now that seeing a semantically (e.g., 'sell') or morphologically (e.g., 'dealer') related word makes it easier to then identify a given target (e.g., 'deal'; Feldman (2000); Forster et al. (1987); Grainger et al. (1991); Neely (1977); Rastle et al. (2000)). Exactly because the lexical system is bombarded with words in very rapid succession during sentence reading, lexical identification should be dominated by this kind of cross - word effects. Just as well as seeing 'nice' in the middle of a black screen would make it quicker to identify the word 'kind' in isolation, reading the former word should make it easier to then recognize the latter when they lie close within the same sentence. In other words, there should be extensive cross - word priming during sentence reading. This is the phenomenon we investigate in this paper, to start filling the gap between individual word identification and sentence reading.

Although from a rather different perspective, lexical dynamics during sentence reading were of course investigated in previous work. For example, a number of studies have addressed semantic priming in sentences, and yielded mixed results - while some have reported facilitation (e.g., Blank and Foss, 1978; Van Petten et al., 1997), others have found that lexical priming is easily overridden by message-level factors, such as discourse context and predictability (e.g., Duffy et al., 1989; Morris, 1994; Morris and Folk, 1998; Traxler et al., 2000). For example, Morris (1994) reported savings in the identification of a target word (e.g., 'moustache') from a related verb prime ('trimmed') in sentences like 'the gardener 
talked as the barber trimmed the moustache', but not in sentences with manipulated semantic relationship between the subject and the verb, like 'the gardener talked to the barber and trimmed the moustache', suggesting that the facilitation of target word identification depends on discourse context.

Morphological effects were also heavily studied in sentence reading (e.g., Barber and Carreiras, 2005; Kos et al., 2010; Kutas and Hillyard, 1980, 1984; Weber and Lavric, 2008). Most of this work, however, is rather difficult to interpret in terms of cross-word priming, given that it is based on morpho-syntactic violation paradigms (e.g., 'the old man eats an apples').

Some morphological congruity effects in naturalistic material were indeed observed, which can be interpreted in terms of cross-word morphological priming. However, these experiments typically involved rather minimalistic environments such as word pairs (e.g., Goodman et al., 1981; Samar and Berent, 1986; West and Stanovich, 1988). For example, they report how the (dis)agreement between an adjective and its following noun (e.g., 'brutta casa' vs. *'brutto casa') influences the processing of the latter, where the introduced disagreement results in e.g., longer response time in word repetition tasks, compared to when noun agreement is not violated (Bates et al., 1996). It is far from clear whether this kind of effects would generalise to content word priming, similar to what is typically investigated in the individual word literature (e.g., dealer-deal, kindness-softness). Also, it is not established whether they would emerge in naturalistic, everyday life sentence reading - without this, we miss the bridge between single word identification and text reading that we are aiming at.

Eye tracking can help us out of the unnaturalistic paradigms that were adopted in previous studies, thus allowing us to assess cross-word priming under more natural reading conditions. In fact, priming may emerge as shorter fixations on words that were preceded by semantically or morphologically congruent words, while participants are simply asked to read sentences for comprehension. At the same time, eye tracking allows us to use the material that is typically adopted in the single word literature. For example, content words that are semantically and/or morphologically congruent are located close to each other in a sentence (e.g., '...forks and spoons...'), so that if the relevant information persists in the lexical system, we should observe savings in the identification time (that is, fixation durations) of the latter word. Quite conveniently, eye tracking would also allow us to estimate the time course of the eventual effects, through a comparison between earlier fixation measures (e.g., first-of-many fixation duration) and later eye movement metrics (e.g., gaze duration). Finally, eye tracking would also allow us to inspect target word skipping, which potentially provides insight into the information that is extracted from parafoveal words.

As far as semantics goes, we are aware of only two studies that adopted a similar paradigm. Carroll and Slowiaczek (1986) focused on how the structure of the sentence would affect lexical processing. They found that priming is influenced by the syntactic structure of the sentence, so that it is observed only when the semantically related prime and target appear in the same clause (e.g., 'The guard saluted the king and the queen in the 
carriage, but they didn't notice'). One issue with this experiment, however, is the lack of control over target word predictability. As observed in, e.g., Otten and Van Berkum (2008), there is widespread prediction of the upcoming word during sentence reading, which makes it difficult to unambiguously attribute Carroll's and Slowiaczek's results to cross-word priming. Facilitation may actually come from the on-line prediction of the target, to which the prime surely contributes as part of the sentence, but which it doesn't determine per se. The interaction between lexical representations in the mental lexicon may not be the driving force behind these results.

A similar issue affects the study of Camblin et al. (2007). These authors did gather data on target word predictability, but mean cloze probability in their semantically congruent condition was very high (averaging .36). Thus, on-line prediction based on sentence context may have played a major role in this experiment too, making unclear the contribution of lexical dynamics.

Things are not entirely clear on the morphological side either. For example, Paterson et al. (2011) investigated how the prior exposure to morphologically related words may influence target word processing. Their prime-target pairs were either semantically transparent (e.g., marshy-marsh), had only an apparent morphological relationship (e.g., secretarysecret), or were morphologically unrelated but as orthographically similar as in the previous conditions (e.g., extract-extra). Priming effects were observed in the semantically transparent pairs, but were absent in the remaining two conditions. This study clearly shows that the observed morphological priming effect is not driven by the prime-target (morpho)orthographic relationship (e.g., Rastle et al., 2004). However, as the authors themselves acknowledge, it is impossible to establish whether the observed effect is morphological in nature or is rather a more general semantic effect, of the sort we would find with words like 'cat' and 'dog' — without such a pure semantic condition, this question cannot be addressed.

In the current study, we build on this previous work and devise a design that unambiguously assesses semantic and morphological cross-word priming during sentence reading. The core idea is quite simple. Primes and targets are embedded into sentences and put close together in a coordinating phrase (e.g., 'Paul entered a room with a table and a chair, which didn't really look like a kitchen'). Their semantic (S) and morphological (M) relationship is then independently manipulated, e.g., 'a table and a chair' $(\mathrm{S}+\mathrm{M}+)$ vs. 'a dog and a chair' $(\mathrm{S}-\mathrm{M}+)$ vs. 'some tables and a chair' $(\mathrm{S}+\mathrm{M}-)$ vs. 'some dogs and a chair' (S-M-). Priming is taken to occur if fixations on the target word ('chair') are shorter after semantically and/or morphologically congruent primes.

This paradigm, as illustrated above based on English, has two main problems though. First, as already pointed out by Paterson et al. (2011), morphological relationship brings about orthographic relationship (plural words share a final $-\mathrm{s}$ ), which makes it difficult to disentangle these two effects, in particular for shorter words. Second, the presence of determiners in the critical bit of the sentence is not ideal, for at least two reasons. Although articles are typically skipped during reading (e.g., Angele and Rayner, 2013), they could potentially attract at least some fixations, which would be difficult to handle- should they 
count as fixations on the target word? Or perhaps they would determine quite some more skipping of the target word itself? Also, and probably more relevant, articles contain morphological information, which would be extracted by the readers (foveally or parafoveally), thus blurring the whole picture - would morphological priming come from the content words or the determiners, or some cross-talk between the two? How does this affect the pattern of results, if it does at all? How would then results be comparable to those emerging from the individual word literature?

Here is where Slovenian, the language that we used for this experiment, turns out to be handy. Slovenian does not use determiners, so that primes and targets would sit alone in the critical coordinating phrase (e.g., 'miza in stol', 'a table and a chair'). Also, Slovenian is inflectionally very reach - it has 6 different cases, 3 different genders, and 3 different grammatical numbers, with noun declension introducing distinct suffixes for different combinations of the three. Nouns can thus be inflected in the same way (i.e., in number and case), but still have orthographically different suffixes (e.g,. 'avtomobil-i', 'cars', plural, nominative; and 'učiteljic-e', 'teachers', plural, nominative,). This peculiarity of the Slovenian language thus enables us to rule out any orthographic contribution to morphological priming.

So, to sum up, we investigate here morphological and semantic cross-word priming during sentence reading. We do this in a natural reading, eye tracking paradigm that, taking advantage of the features of Slovenian, allows us to test these effects with a very ecological paradigm, virtually free of the confounds that affect the data available to date. This, we hope, will start bridging the current gap between the precise and mechanistic theories that we have for single word identification, and how the information extracted from single words is then used to build a complete linguistic message.

\section{Methods}

\subsection{Participants}

44 native Slovenian speakers $(\mathrm{F}=28)$ took part in the study. Their mean age and education was 34.2 (range $=20-60$ ) and 15.5 years (range $=12-18$ ), respectively. They all grew up in a monolingual environment and had normal or corrected-to-normal vision. Participants received an 8, 15 or 20 Euro compensation, depending on the distance travelled in order to reach the place of testing (SISSA, Trieste, Italy, or the University of Ljubljana, Slovenia). They all provided their informed consent to take part in the study before the beginning of the experiment.

\subsection{Materials}

The stimuli set comprised 40 sentences, in which two nouns appeared one after the other, separated by the conjunction in, and (e.g., 'kolesar ni bil pozoren na avto in tovornjak in je zato povzročil nesrečo'; 'the cyclist was not paying attention to a car and a truck and therefore caused an accident'). In our design, the first noun (avto, car, in the example) is the prime word, while the second noun (tovornjak, truck, in the example) is the target word. 
These sentences appeared in 4 different conditions, where primes and targets were (i) related in meaning, and inflected in the same grammatical number (avto-tovornjak, (a) car-(a) truck); (ii) related in meaning, but not inflected in the same grammatical number (avte-tovornjak, (some) cars-(a) truck); (iii) unrelated in meaning, and inflected in the same grammatical number (lužo-tovornjak, (a) puddle-(a) truck); (iv) unrelated in meaning, and not inflected in the same grammatical number (luže-tovornjak, (some) puddles-(a) truck). Both the carrier sentences and the target words were kept identical across conditions; only the prime varied, to determine morphological and/or semantic relatedness in a crossed, 2-by-2 design.

While constructing the sentences, we took a series of measures to guarantee a fair assessment of cross-word priming. First, the prime and the target word always appeared within the same syntactic clause (Carroll and Slowiaczek, 1986; Morris and Folk, 1998). Second, as anticipated above, primes and targets never shared the same orthographic suffix, and only shared the same final letter in 9 cases (5.6\% of the stimuli set), so as to rule out any substantial contribution from form priming. As mentioned above, this is easily obtained in Slovenian through the use of prime and target nouns with different gender (e.g., brisač- $i$, towels, and ležalnik-a, deckchairs, are both dual nouns in the nominative case). Furthermore, target words never appeared in either clause or sentence final position, and were never followed by a comma - these conditions may in fact elicit wrap-up effects, with longer fixations depending on syntactic and/or semantic integration (Warren et al., 2009). Finally, we also controlled the position of the prime and the target word on the screen - they never appeared as the first or the last words in a line.

Primes and target features are illustrated in Table 1. They were matched as closely as possible for length and frequency. This latter was taken from the Slovenian corpus Gigafida. Sentences were 12 to 20 words long (mean=15.6), and included 63 to 138 characters overall (mean=92.3). The prime words came 19 to 62 characters into the sentence (mean=36.7). 25 sentences were displayed in two lines of text, whereas 15 occupied three lines of text on the screen.

Table 1: Frequency and length of our stimuli across conditions. We report means and SDs. Frequency is taken from the Slovenian corpus Gigafida, log transformed, and based on word form.

\begin{tabular}{lccccc}
\hline & $\begin{array}{c}\text { S+M+ } \\
\text { prime }\end{array}$ & $\begin{array}{l}\text { S+M- } \\
\text { prime }\end{array}$ & $\begin{array}{l}\text { S-M+ } \\
\text { prime }\end{array}$ & $\begin{array}{c}\text { S-M- } \\
\text { prime }\end{array}$ & Target \\
\hline $\begin{array}{c}\text { Frequency } \\
\text { Length }\end{array}$ & $1.46(0.54)$ & $1.35(0.53)$ & $1.34(0.58)$ & $1.36(0.52)$ & $1.32(0.50)$ \\
\hline
\end{tabular}

$\mathrm{S}+/-$ : semantically congruent/incongruent; $\mathrm{M}+/-$ : morphologically congruent/incongruent.

In order to unambiguously asses the cross-word priming effects and also to prevent an ex- 
cessive skipping of the target words, we made sure that these latter were not too predictable. A cloze probability task was set up (Kutas and Hillyard, 1984) with all the sentences that were then used in the experiment proper. A separate sample of 80 participants $(\mathrm{F}=54$; mean age $=32.38$; age range $=20-65$ ), none of whom took part in the eye tracking experiment, were presented with the experimental sentences up to the pre-target word and were asked to complete them with the first word that came to mind. Because of our design, each target was anticipated by four different primes, in four otherwise identical sentences (e.g., Kolesar ni bil pozoren na avto/avte/lužo/luže in tovornjak ...). To make sure that target predictability was similar (and low) across conditions, all of these four sentences were tested in the Cloze Probability task, using a Latin Square design (each participant was presented with only one item in each sentence quadruplet, rotated across conditions). In the final stimuli set, no target word has a cloze probability higher than .2, with means and SDs across conditions as illustrated in Table 2.

Table 2: Mean (SD) cloze probability across conditions.

\begin{tabular}{lcccc}
\hline & $\mathbf{S}+/ \mathbf{M}+$ & $\mathbf{S}+/ \mathbf{M}-$ & $\mathbf{S}-/ \mathbf{M}+$ & $\mathbf{S}-/ \mathbf{M}-$ \\
\hline Cloze probability & $.05(.05)$ & $.06(.07)$ & $.01(.03)$ & $.01(.03)$ \\
\hline
\end{tabular}

$\mathrm{S}+/-:$ semantically congruent/incongruent; $\mathrm{M}+/-:$ morphologically congruent/incongruent.

We also tested the strength of the semantic relatedness between the target words and their primes. A further separate sample of 21 participants $(\mathrm{F}=12$; mean age $=38$; age range $=25-58$ ), none of whom took part in the eye tracking experiment or the cloze probability task, was asked to rate each prime-target pair for similarity in meaning on a 1-to-5 scale (1, not similar at all; 5, very similar). Because, again, each target was associated with two different primes (primes were tested only in one morphological form here), participants were rotated over conditions (related vs. unrelated) in a Latin Square design. The results of this pre-test are illustrated in Figure 1, and show that semantically congruent primes were rated as substantially more related to their targets than semantically incongruent ones, consistently across targets (mean and SD are 3.38 and 0.54, respectively, for the congruent condition; and 1.74 and 0.42 for the incongruent condition).

\subsection{Procedure}

Participants were calibrated with a standard 9-point grid. Calibration was automatically checked with the fixation point before the beginning of each trial, and was repeated when necessary.

Participants were instructed to read silently the sentences at their own pace. On roughly $30 \%$ of the trials, sentence reading was followed by a $2 \mathrm{AFC}$ comprehension question, to ensure that participants were actually reading and understanding the sentences. 


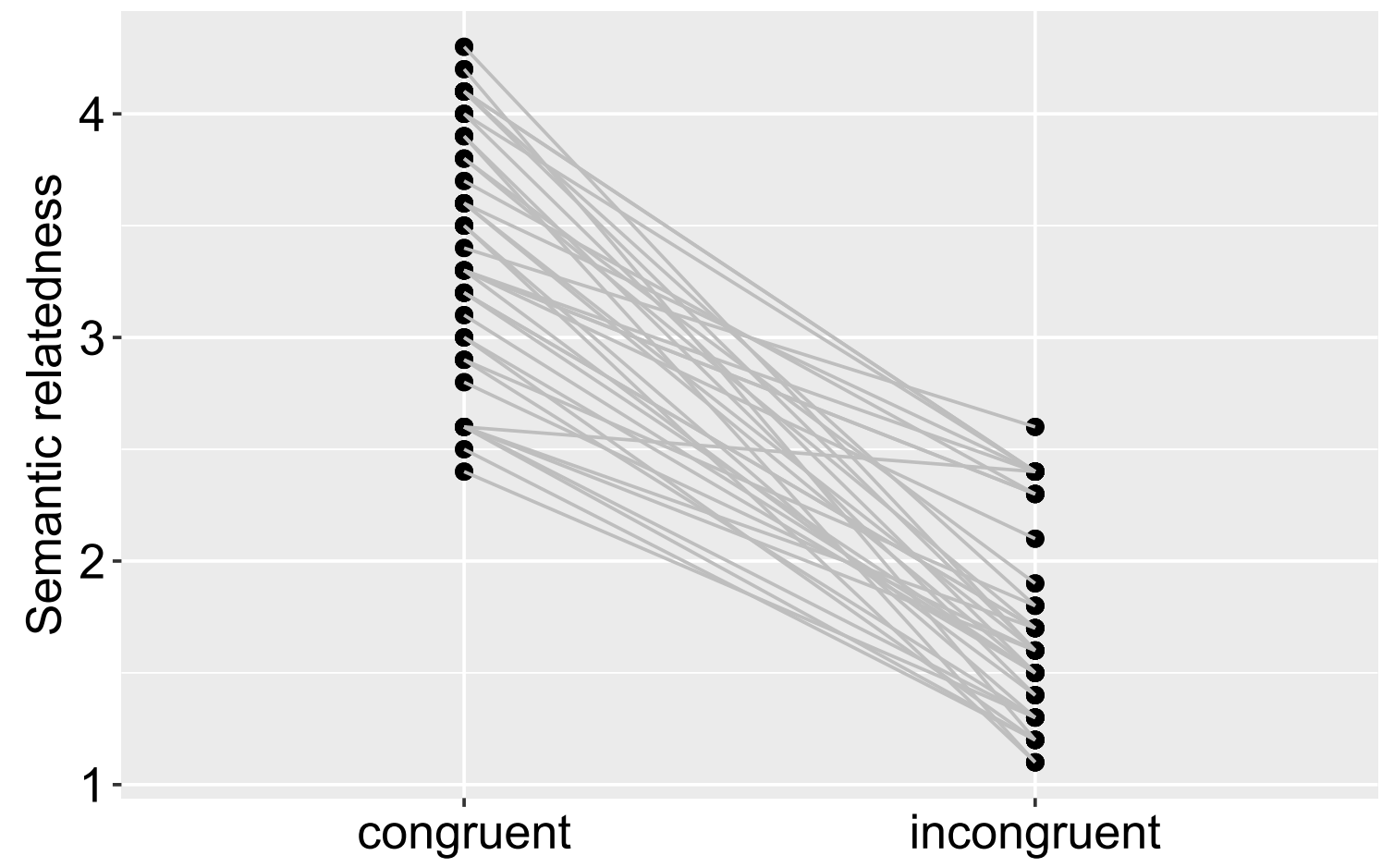

Figure 1: Semantic relatedness in our Congruent (left) and Incongruent (right) pairs. Grey lines connect corresponding prime, those that were used with the same target.

The trial timeline was as follows. A fixation point appeared on the screen for $250 \mathrm{~ms}$. Then the full sentence was displayed on the screen, and remained visible until the participants pressed the space bar, indicating that they have finished reading. This led to the presentation of either the fixation point of the following trial, or the comprehension question.

In order to reduce eventual repetition effects, each participant was presented with each sentence in only two out of the four experimental conditions, with a Latin Square design rotation. Each session was divided in two blocks, with the same two sentences never being part of the same block. To further minimize repetition effects, and also to reduce participants' awareness of the goal and the structure of the experiment, each block also included 60 filler sentences, which were not part of the experimental design and were not analyzed. The critical sentences were arranged across blocks in such a way that each block included 10 sentences per condition. Overall, each participant read a total of 200 sentences, in two separate blocks of 100 each. The experiment lasted about 50 minutes.

\subsection{Apparatus}

Participants sat $56 \mathrm{~cm}$ from the computer screen where the stimuli were displayed. Their head was stabilized through a chin rests. An SR Eyelink $1000+$ was used to record participants' eye movements, at a sampling rate of $1000 \mathrm{~Hz}$. Eye movements were recorded from the dominant eye only. 


\subsection{Data analysis}

Two interest areas were created for each sentence around the prime and the target words, using SR Research Data Viewer (SR Research Ltd., Kanata, ON, Canada). Trials with large gaze drifts were discarded.

Because we were interested in exploring the time course of the eventual cross-word priming, we analysed a number of target fixation metrics-first-of-many (FoM) fixation duration, first fixation duration, single fixation duration, gaze duration and total looking time. Moreover, in order to check for potential parafoveal priming, we also analysed target skipping and target-on-prime facilitation.

Data were analyzed with R (R Development Core Team, 2008), using Rstudio (RStudioTeam, 2016) and the lme4 package (Bates et al., 2015) for fitting (generalised) linear mixed models. Model estimates and effect sizes were obtained using the package Effects (Fox and Hong, 2009). Continuous dependent variables were all log transformed in order to approximate a normal distribution and to achieve symmetrically distributed model residuals. They were modelled as a function of semantic and syntactic congruency, with subjects and target words as random intercepts. Statistical significance was checked both for model parameters and for predictors overall. Effects were checked for their dependence on outliers following Baayen (2008) - models were re-run after excluding data points whose standardised residuals were larger than 2.5 in absolute value. There were no effects that would be significant only with (or without) outliers; reported here are the results of the models including all data points.

All data and analysis scripts are available to the reader here.

\section{Results}

All participants responded correctly to at least $95 \%$ of the comprehension questions (overall mean $=98 \%, \mathrm{SD}=1.64 \%$ ), which suggests that they performed the task appropriately and understood the sentences very well. One participant was however excluded from the analysis because of her/his abnormally long fixations - her/his grand average gaze duration on the target words was $807.6 \mathrm{~ms}$, more than $300 \mathrm{~ms}$ slower than the next slowest participant.

The overall descriptive statistics for the variables that we considered in the analyses are reported in Table 3.

Starting from the earliest time point where priming could be reasonably expected, we checked whether target skipping might be influenced by the nature of the primes. Since the decision to skip the target word must be made prior to the target being fixated, this type of priming would be based on parafoveal information. Our dataset displays a relatively small number of target word skips $(\mathrm{n}=151)$, which amounts to $\sim 5 \%$ of all trials. There is a significant effect of semantic congruency, $F(1,2687)=4.05, p=.04$ (the corresponding model parameter is also very close to significance, $z=1.74, p=.08) .95 \%$ confidence intervals (CI) for target skipping probablity are [.025 - .059] and [.015 - .039] for semantically congruent and incongruent primes, respectively. Pointwise model predictions reveal that targets are 
Table 3: Means (and standard deviations) across conditions for the eyetracking metrics that we considered in this paper. Statistics are reported in $\mathrm{ms}$ or as proportions (for skipping rate), and are based on unaggregated data. Note: Skip, skipping rate; Prime, prime fixation duration (which tracks target-on-prime effects); FoM, first-of-many fixation duration; Single, single fixation duration; First, overall first fixation duration; Gaze, gaze duration; TLT, total looking time; $\mathrm{S}+/-$, semantically congruent/incongruent; $\mathrm{M}+/-$, morphologically congruent/incongruent.

\begin{tabular}{ccccc}
\hline & $\mathrm{S}+\mathrm{M}+$ & $\mathrm{S}+\mathrm{M}-$ & $\mathrm{S}-\mathrm{M}+$ & $\mathrm{S}-\mathrm{M}-$ \\
\hline Skip & $.066(.249)$ & $.067(.251)$ & $.048(.214)$ & $.041(.199)$ \\
Prime & $391(350)$ & $417(367)$ & $414(356)$ & $395(339)$ \\
FoM & $228(110)$ & $232(135)$ & $238(149)$ & $235(133)$ \\
Single & $243(145)$ & $237(126)$ & $256(150)$ & $249(145)$ \\
First & $237(134)$ & $235(129)$ & $249(149)$ & $243(140)$ \\
Gaze & $312(189)$ & $319(196)$ & $345(194)$ & $334(196)$ \\
TLT & $441(312)$ & $453(304)$ & $558(379)$ & $534(368)$ \\
\hline
\end{tabular}

.014 more likely to be skipped if anticipated by a semantically related word. In contrast, our data suggest no effect of morphological congruency, $F(1,2687)=.01, p=.92$, nor any interaction between semantic and morphological relatedness, $F(1,2687)=.21, p=.64$. The model predictions for each design cell are illustrated in Figure 2.

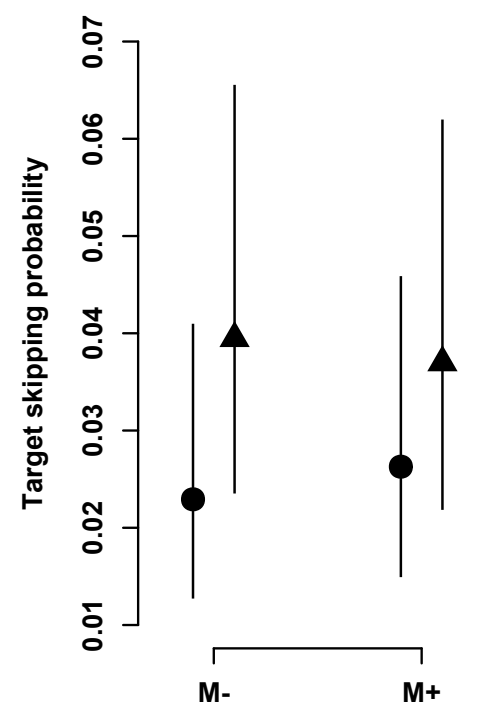

Figure 2: LMM estimates for proportions of target word skip. Error bars represent 95\% CIs. Note: semantically incongruent/congruent; M-/ + , morphologically incongruent/congruent. 
We also assessed priming based on parafoveal information in the form of a target-onprime effect - essentially, we checked whether first-pass fixations on primes were shorter when the following target words were semantically and/or morphologically related ${ }^{1}$. This phenomenon would belong to the class of the hotly debated Parafoveal-on-Foveal (PoF) effects (e.g., Just and Carpenter, 1983; Henderson and Ferreira, 1993; Inhoff et al., 2000). Our data show no suggestion of any semantic, $F(1,2549)=.03, p=.85$, morphological, $F(1,2549)=.10, p=.75$, or interaction effect, $F(1,2549)=.10, p=.75$.

First-of-many (FoM) fixation durations do not reveal any effect of semantic congruity, $F(1,946)=.61, p=.43$, morphological congruity, $F(1,946)=.01, p=.92$, or interaction between the two, $F(1,946)=.06, p=.80$. The overall effect size is $5.9 \mathrm{~ms}$ for semantic priming and $.7 \mathrm{~ms}$ for morphological priming. Figure 3 a presents the model-based estimates for FoM. Interestingly, the lack of priming is not due to the skipping of the prime words - this only happened in $3.4 \%$ of the trials and, unsurprisingly, the model where these trials were excluded yields exactly the same results (all $\mathrm{p}>.61$ ).

The same pattern emerges in single fixation duration, and in the duration of first fixations overall-no priming effect seems to emerge whatsoever in these metrics (all $p>.20$ and all effect sizes are below $<6.9 \mathrm{~ms}$ ).

Gaze duration (GD), instead, reveals a solid semantic priming effect, $F(1,2535)=5.12$, $p=.02$ (the corresponding model parameter is close to significance too, $t(2535)=<1.17$, $p=.12$ ), with no morphological priming, $F(1,2535)=.39, p=.53$, nor interaction between semantic and morphological congruity, $F(1,2535)=.36, p=.54$. The estimated effect size is $18.6 \mathrm{~ms}$ for semantic priming and $5.1 \mathrm{~ms}$ for morphological priming. Figure 3b presents the model-based estimates for the four design cells. When only trials where the prime was fixated are taken into consideration, the semantic effect becomes even stronger, $F(1,2420)=8.40$, $p=.004$ (model parameter, $z=1.54, p=.06$ ). The morphological effect and the interaction between semantic and morphological relationships remains far from significance (all $\mathrm{p}>.47$ )

The same pattern emerges in the total viewing time - a solid semantic priming effect emerges, $F(1,2535)=17.63, p<.001$ (model parameter, $t(2535)=-2.50, p=.006$ ), with no morphological priming $F(1,2535)=.26, p=.6$, nor interaction between semantic and morphological congruity $F(1,2535)=.44, p=.5$.

\section{Discussion}

Building on similar previous paradigms and taking advantage of some handy features of the Slovenian language, we investigated semantic and morphological cross-word priming during natural sentence reading. Semantic facilitation emerged clearly, both in the skipping

\footnotetext{
${ }^{1}$ Please note that, because the experiment was not designed specifically for testing this effect, priming is assessed across different targets here. Results should thus be taken with some caution.
} 


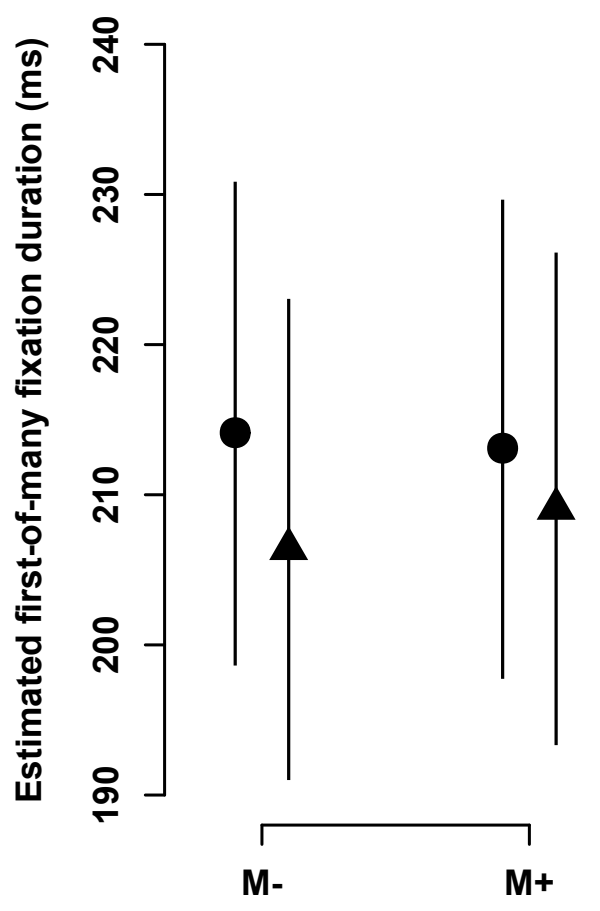

(a)

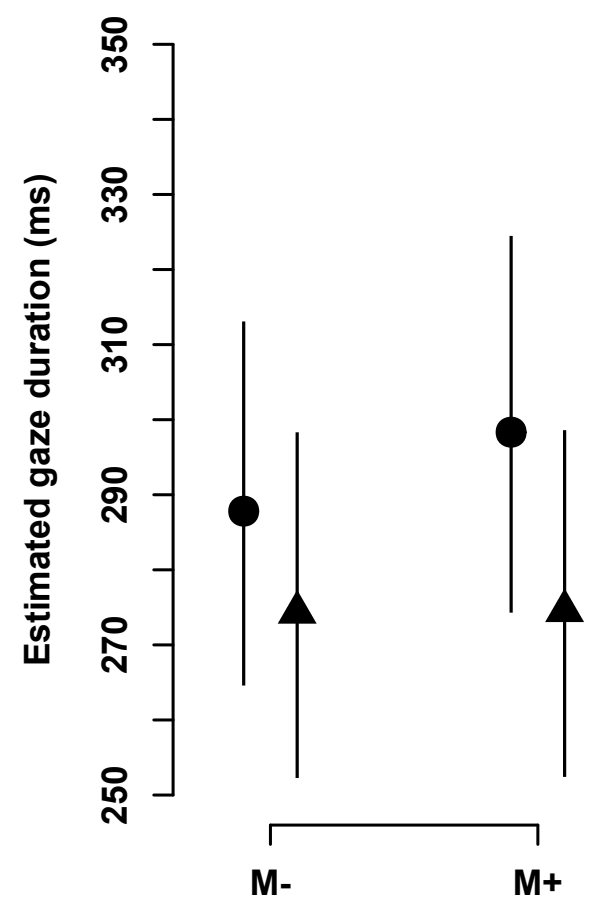

(b)

Figure 3: LMM estimates for FoM fixation durations (panel a) and GD (panel b) on the target words. Error bars represent 95\% CIs. Note: - / , semantically incongruent/congruent; M-/+, morphologically incongruent/congruent.

rate and in relatively late indexes of target fixation. This effect was not modulated by morphological agreement, nor we observed any morphological priming in the first place. Importantly, all these results emerged in a very ecological, natural sentence reading paradigm, thus revealing lexical dynamics as they likely emerge in our everyday reading experience. Moreover, these data extends our theoretical knowledge by showing us what happens when the visual word identification system is engaged with several words in a short amount time.

Our results confirm previous observations of cross-word semantic priming in natural sentence reading (Carroll and Slowiaczek, 1986; Camblin et al., 2007). Importantly, the current experiment allows to rule out word predictability in the sentence context as a source for this priming. Despite target words were barely predictable, and not differently so after related and unrelated primes, looking times were shorter on word $\mathrm{N}$ when word $\mathrm{N}-1$ had a similar meaning. We can thus attribute the observed effect to the residual activation in 
the lexical system from word N-1 when a semantically related word $\mathrm{N}$ comes to the stage. This clearly indicates that there is no cross--word reset in the lexical system during sentence reading.

Whether this means that neighbouring words are taken up by the system simultaneously (Snell et al., 2018; Engbert et al., 2005) or in rapid succession (Reichle et al., 2006), we cannot tell based on these data. The former hypothesis, however, would open a very interesting question - how does the lexical system keep track of information coming from different words? This is a nice challenge for computational models of reading and eye movements, which has only started to be taken up (see, for example, Snell et al., 2018)

The time course of this semantic priming effect is also interesting. Facilitation emerged first in the skipping rate. This is perhaps not particularly surprising, given that the decision of whether to skip word $\mathrm{N}$ must obviously come while word N-1 is fixated; thus, the fact that information on word N-1 plays a role here is hardly unanticipated. But then, semantic priming did not emerge in earlier indexes of target fixation, such as FoM; and then 'came back' in later measures, such as GD. This contrast between FoM and GD would suggest a qualitative difference between the computations that determine overall first-pass looking time, which would be sensitive to word N-1 meaning; and those behind the earlier decision of whether to spend a second fixation on the foveal word, which would instead be independent of word N-1 semantics. Whatever precise interpretation one adopts for this time course, one thing is clear - it is not a matter of a mere activation decay for word $\mathrm{N}-1$, or otherwise we would have observed stronger priming in earlier eye tracking indexes. Rather, these data suggest a dynamic, goal-specific cross-word processing in the lexical system during text reading, which is another interesting challenge for general theories of eye movements and visual word identification.

We were not able to observe any morphological priming instead. This better qualifies the evidence provided by previous experiments (e.g., Paterson et al., 2011) — when semantics and morphology are manipulated independently, the latter does not seem to give rise to cross-word processing savings during sentence reading. These data would suggest a reinterpretation of Paterson et al. (2011) results in terms of semantic similarity; 'marshy' would prime 'marsh' because these words have close meanings, not because they are morphological relatives. This would also explain the lack of any effect in opaque pairs like 'secretary' and 'secret' in the same experiment.

A note of caution is in order here, though. Especially in the individual word literature, morphological priming is typically addressed though shared stems or affixes, that is, in pairs like dealer-deal, or kindness-softness (e.g., Crepaldi et al., 2016; Rastle et al., 2004; Marslen-Wilson, 2007). Here, instead, primes and targets shared an abstract morphological inflection, which was denoted by different affixal, orthographic realizations (e.g., avtomobil$a$, (two) cars, and mačk-i, (two) cats). This approach allows ruling out any orthographic (or phonological) contribution to morphological effects, which is why we adopted it. However, it may also justify the discrepancy between what we find here and the vast individual word priming literature showing solid morphological facilitation (e.g., Marslen-Wilson et al., 1994; 
Feldman, 2000; Rastle et al., 2000; Gonnerman et al., 2007).

Another very important insight brought about by cross-word priming is the stark contrast between semantic and morphological processing during sentence reading. Not only semantic facilitation emerges while morphological priming does not, but also we were unable to see any interaction between the two players - the semantic effect was not affected by whether primes and targets were inflected alike, nor the morphological effect was modulated by semantic similarity. This may suggest that abstract morphological agreement is processed, at least in part, outside of the (semantic) lexicon; and is reminiscent of theories supporting distinct lexical-semantic and morphological systems (e.g., Mcbride-Chang et al., 2008; Ramirez et al., 2014). Whatever theoretical interpretation one may want to adopt here however, these data are quite clear in showing a more locally encapsulated morphological system, which seems to prevent processing spillover between neighbouring words.

Finally, the cross-word semantic priming observed here in the skipping rate of the target word joins the club of recent papers showing semantic information uptake in the parafovea (e.g., Schotter, 1996; Veldre and Andrews, 2016b,a; Hohenstein and Kliegl, 2014). This adds to a long-lasting debate, which has been a major battlecamp between serial and parallel models of eye movements during reading (e.g., Reichle et al., 1998). The data presented here seem to favour the latter, as semantic processing of word $\mathrm{N}+1$ would be difficult to justify in the former camp (but see Schotter et al., 2014, for an argument in this direction).

In addition to providing theoretical insight, the data described in the present paper open a few interesting questions, which the novel paradigm established here may help addressing. First, an important next step would be to study cross-word priming with different types of similarities between the prime and the target, such as case/gender agreement or orthographic similarity. This latter in particular may prove particularly interesting. Individual word priming suggests that orthographic overlap between the prime and the target may trigger lexical competition (e.g., Crepaldi et al., 2016; Davis and Lupker, 2006), which asks the question of whether we would see inhibitory cross-word priming between orthographically similar words.

Another interesting issue is related to the distance between primes and targets. In our sentences, they were only separated by a short, high-frequency conjunction word; and always sat within the same coordinating phrase. How much lag is cross-word priming able to overcome? And how would syntax play out here? We have observed that morphological inflection does not seem to affect semantic facilitation during sentence reading; would it be the same for perhaps more prominent morpho-syntactic factors such as phrase boundaries, or word movement traces?

\section{References}


Adelman, J. S., Oct. 2011. Letters in time and retinotopic space. Psychol. Rev. 118 (4), 570-582.

Adelman, J. S., Marquis, S. J., Sabatos-DeVito, M. G., Dec. 2010. Letters in words are read simultaneously, not in left-to-right sequence. Psychol. Sci. 21 (12), 1799-1801.

Angele, B., Rayner, K., Mar. 2013. Processing the in the parafovea: are articles skipped automatically? J. Exp. Psychol. Learn. Mem. Cogn. 39 (2), 649-662.

Baayen, R. H., Mar. 2008. Analyzing Linguistic Data: A Practical Introduction to Statistics using R. Cambridge University Press.

Barber, H., Carreiras, M., Jan. 2005. Grammatical gender and number agreement in spanish: an ERP comparison. J. Cogn. Neurosci. 17 (1), 137-153.

Bates, D., Mächler, M., Bolker, B., Walker, S., Oct. 2015. Fitting linear Mixed-Effects models using lme4. J. Stat. Softw. 67 (1), 1-48.

Bates, E., Devescovi, A., Hernandez, A.and Pizzamiglio, L., 1996. Gender priming in italian. Perception and Psychophysics 85 (1), 992-1004.

Blank, M. A., Foss, D. J., Nov. 1978. Semantic facilitation and lexical access during sentence processing. Mem. Cognit. 6 (6), 644-652.

Camblin, C., Gordon, P. C., Swaab, T. Y., 2007. The interplay of discourse congruence and lexical association during sentence processing: Evidence from ERPs and eye tracking. J. Mem. Lang. 56 (1), 103-128.

Carroll, P., Slowiaczek, M. L., Nov. 1986. Constraints on semantic priming in reading: a fixation time analysis. Mem. Cognit. 14 (6), 509-522.

Coltheart, M., Rastle, K., Perry, C., Langdon, R., Ziegler, J., Jan. 2001. DRC: a dual route cascaded model of visual word recognition and reading aloud. Psychol. Rev. 108 (1), 204-256.

Crepaldi, D., Hemsworth, L., Davis, C. J., Rastle, K., 2016. Masked suffix priming and morpheme positional constraints. Q J Exp Psychol 69 (1), 113-128.

Davis, C. J., Jul. 2010. The spatial coding model of visual word identification. Psychol. Rev. 117 (3), $713-758$.

Davis, C. J., Lupker, S. J., Jun. 2006. Masked inhibitory priming in english: evidence for lexical inhibition. J. Exp. Psychol. Hum. Percept. Perform. 32 (3), 668-687.

Duffy, S. A., Henderson, J. M., Morris, R. K., 1989. Semantic facilitation of lexical access during sentence processing. J Exp Psychol Learn Mem Cogn 15 (5), 791-801.

Engbert, R., Nuthmann, A., Richter, E. M., Kliegl, R., 2005. SWIFT: A dynamical model of saccade generation during reading. Psychol. Rev. 112 (4), 777-813.

Feldman, L. B., Nov. 2000. Are morphological effects distinguishable from the effects of shared meaning and shared form? J. Exp. Psychol. Learn. Mem. Cogn. 26 (6), 1431-1444.

Forster, K. I., Davis, C., Schoknecht, C., Carter, R., 1987. Masked priming with graphemically related forms: repetition or partial activation? The Quarterly Journal of Experimental Psychology 39, $211-251$.

Forster, K. I., Veres, C., Mar. 1998. The prime lexicality effect: form-priming as a function of prime awareness, lexical status, and discrimination difficulty. J. Exp. Psychol. Learn. Mem. Cogn. 24 (2), $498-$ 514.

Fox, J., Hong, J., 2009. Effect displays inrfor multinomial and Proportional-Odds logit models: Extensions to theeffectspackage. J. Stat. Softw. 32 (1).

Gonnerman, L. M., Seidenberg, M. S., Andersen, E. S., 2007. Graded semantic and phonological similarity effects in priming: Evidence for a distributed connectionist approach to morphology. Journal of Experimental Psychology: General 136, 323-345.

Goodman, G. O., McClelland, J. L., Gibbs, Jr, R. W., Nov. 1981. The role of syntactic context in word recognition. Mem. Cognit. 9 (6), 580-586.

Grainger, J., Colé, P., Segui, J., 1991. Masked morphological priming in visual word recognition. Journal of Memory and Language 30, 370-384.

Henderson, J. M., Ferreira, F., Jun. 1993. Eye movement control during reading: fixation measures reflect foveal but not parafoveal processing difficulty. Can. J. Exp. Psychol. 47 (2), 201-221.

Hohenstein, S., Kliegl, R., 2014. Semantic preview benefit during reading. Journal of Experimental Psychology: Learning, Memory, and Cognition 40 (1), 166-190.

Inhoff, A. W., Radach, R., Starr, M., Greenberg, S., 2000. Allocation of Visuo-Spatial attention and saccade 
programming during reading. In: Reading as a Perceptual Process. pp. 221-246.

Just, M., Carpenter, P., 1983. What your eyes do while your mind is reading. In: Rayner, K. (Ed.), Eye Movements in Reading: Perceptual and Language Processes. Academic press, New York, pp. 275 - 305.

Kos, M., Vosse, T., van den Brink, D., Hagoort, P., Dec. 2010. About edible restaurants: Conflicts between syntax and semantics as revealed by ERPs. Front. Psychol. 1, 222.

Kutas, M., Hillyard, S. A., Jan. 1980. Reading senseless sentences: brain potentials reflect semantic incongruity. Science 207 (4427), 203-205.

Kutas, M., Hillyard, S. A., 1984. Brain potentials during reading reflect word expectancy and semantic association. Nature 307 (5947), 161-163.

Marelli, M., Amenta, S., Crepaldi, D., 2015. Semantic transparency in free stems: The effect of OrthographySemantics consistency on word recognition. Q. J. Exp. Psychol. 68 (8), 1571-1583.

Marslen-Wilson, W., Tyler, L. K., Waksler, R., Older, L., 1994. Morphology and meaning in the english mental lexicon. Psychol. Rev. 101 (1), 3-33.

Marslen-Wilson, W. D., 2007. Morphological processes in language comprehension. In: Gaskell, M. G. (Ed.), The 264 Oxford Handbook of Psycholinguistics. Oxford, pp. 175 - 194.

Mcbride-Chang, C., Tardif, T., Cho, J.-R., Shu, H., Fletcher, P., Stokes, S. F., Wong, A., Leung, K., 2008. What's in a word? morphological awareness and vocabulary knowledge in three languages. Appl. Psycholinguist. 29 (03).

Morris, R. K., Jan. 1994. Lexical and message-level sentence context effects on fixation times in reading. J. Exp. Psychol. Learn. Mem. Cogn. 20 (1), 92-103.

Morris, R. K., Folk, J. R., Nov. 1998. Focus as a contextual priming mechanism in reading. Mem. Cognit. 26 (6), 1313-1322.

Neely, J. H., 1977. Semantic priming and retrieval from lexical memory: Roles of inhibitionless spreading activation and limited-capacity attention. Journal of Experimental Psychology: General 106, 226-254.

Otten, M., Van Berkum, J. J. A., 2008. Discourse-Based word anticipation during language processing: Prediction or priming? Discourse Process. 45 (6), 464-496.

Paterson, K. B., Alcock, A., Liversedge, S. P., 2011. Morphological priming during reading: Evidence from eye movements. Lang. Cogn. Process. 26 (4-6), 600-623.

Pelli, D. G., Tillman, K. A., Freeman, J., Su, M., Berger, T. D., Majaj, N. J., Oct. 2007. Crowding and eccentricity determine reading rate. J. Vis. 7 (2), 20.1-36.

Perry, C., Ziegler, J. C., Zorzi, M., 2010. Beyond single syllables: Large-scale modeling of reading aloud with the connectionist dual process (CDP ) model. Cogn. Psychol. 61 (2), 106-151.

Ramirez, G., Walton, P., Roberts, W., Jan. 2014. Morphological awareness and vocabulary development among kindergartners with different ability levels. J. Learn. Disabil. 47 (1), 54-64.

Rastle, K., Davis, M. H., Marslen-Wilson, W. D., Tyler, L. K., 2000. Morphological and semantic effects in visual word recognition: A time-course study. Lang. Cogn. Process. 15 (4-5), 507-537.

Rastle, K., Davis, M. H., New, B., 2004. The broth in my brother's brothel: Morpho-orthographic segmentation in visual word recognition. Psychonomic Bulletin Review 11 (6), 1090-1098.

Reichle, E. D., Pollatsek, A., Fisher, D. L., Rayner, K., 1998. Toward a model of eye movement control in reading. Psychol. Rev. 105 (1), 125-157.

Reichle, E. D., Pollatsek, A., Rayner, K., 2006. E-Z reader: A cognitive-control, serial-attention model of eye-movement behavior during reading. Cogn. Syst. Res. 7 (1), 4-22.

Reilly, R., Radach, R., 2006. Some empirical tests of an interactive activation model of eye movement control in reading. Cognitive Systems Research 7, 34-55.

RStudioTeam, 2016. Rstudio: Integrated development for r. rstudio, inc., boston, ma. http://www.rstudio. com/, accessed: 2018-07-05.

Samar, V. J., Berent, G. P., Jul. 1986. The syntactic priming effect: evoked response evidence for a prelexical locus. Brain Lang. 28 (2), 250-272.

Schotter, E. R., 1996. Synonyms provide semantic preview benefit in english. Journal of Memory and Language 69, 619-633.

Schotter, E. R., Reichle, E. D., Rayner, K., 2014. Rethinking parafoveal processing in reading: serial- 
attention models can explain semantic preview benefit and $n+2$ preview effects. Visual Cognition 22 (3), 309-333.

Snell, J., van Leipsig, S., Grainger, J., Meeter, M., 2018. Ob1-reader: A model of word recognition and eye movements in text reading. Psychological Review.

Traxler, M. J., Foss, D. J., Seely, R. E., Kaup, B., Morris, R. K., Nov. 2000. Priming in sentence processing: intralexical spreading activation, schemas, and situation models. J. Psycholinguist. Res. 29 (6), 581-595.

Van Petten, C., Weckerly, J., McIsaac, H. K., Kutas, M., 1997. Working memory capacity dissociates lexical and sentential context effects. Psychol. Sci. 8 (3), 238-242.

Veldre, A., Andrews, S., 2016a. Parafoveal preview benefit in sentence reading: Independent effects of plausibility and orthographic relatedness. Psychonomic Bulletin Review 24 (2), 519-528.

Veldre, A., Andrews, S., 2016b. Semantic preview benefit in english: Individual differences in the extraction and use of parafoveal semantic information. Journal of Experimental Psychology: Learning, Memory and Cognition $42(6), 837-854$.

Warren, T., White, S. J., Reichle, E. D., Apr. 2009. Investigating the causes of wrap-up effects: evidence from eye movements and E-Z reader. Cognition 111 (1), 132-137.

Weber, K., Lavric, A., 2008. Syntactic anomaly elicits a lexico-semantic (n400) ERP effect in the second language but not the first. Psychophysiology 45 (6), 920-925.

West, R. F., Stanovich, K. E., Jul. 1988. How much of sentence priming is word priming? Bull. Psychon. Soc. 26 (1), 1-4.

Xu, J., Taft, M., 2014. Solely soles: Inter-lemma competition in inflected word recognition. J. Mem. Lang. $76,127-140$. 